Short Report

\title{
Sipuleucel-T: Autologous Cellular Immunotherapy for Men with Asympto- matic or Minimally Symptomatic Metastatic Castrate Resistant Prostate Cancer
}

\author{
Robert B. Sims ${ }^{凶}$ \\ Dendreon Corporation, Seattle, WA, USA
}

$\triangle$ Corresponding author: Robert B. Sims, MD, Dendreon Corporation, 3005 First Avenue, Seattle, WA 98121. TEL: (206) 829-1457; rsims@dendreon.com.

(c) Ivyspring International Publisher. This is an open-access article distributed under the terms of the Creative Commons License (http://creativecommons.org/ licenses/by-nc-nd/3.0/). Reproduction is permitted for personal, noncommercial use, provided that the article is in whole, unmodified, and properly cited.

Received: 2011.04.29; Accepted: 2011.06.03; Published: 2011.06.08

\begin{abstract}
Sipuleucel $\mathrm{T}$ is an autologous cellular immunotherapy designed to stimulate an immune response in men diagnosed with asymptomatic or minimally symptomatic metastatic castrate resistant (hormone refractory) prostate cancer. Sipuleucel T improves overall survival and provides an additional treatment option for this patient population.
\end{abstract}

Key words: sipuleucel-T, metastatic castrate resistant prostate cancer (mCRPC)

Prostate cancer is a major health concern. It is the second leading cause of death from cancer among males in most western countries. Localized prostate cancer may be cured with surgery or radiation therapy, but unfortunately the disease recurs in up to $30 \%$ of patients. Disease recurrence is often noted by a progressive rise in serum prostate specific antigen (PSA). Hormonal therapy in the form of androgen-deprivation therapy is typically utilized in this setting, but despite initial control the disease eventually progresses to castrate resistant prostate cancer.

Sipuleucel-T is an autologous cellular immunotherapy designed to stimulate an immune response to prostate cancer. It is approved by the United States Food and Drug Administration for the treatment of asymptomatic or minimally symptomatic metastatic castrate resistant (hormone refractory) prostate cancer (mCRPC). The clinical development of sipuleucel-T in mCRPC was based on preclinical demonstration of lymphocytic infiltrates in rat prostate tissue following administration of antigen presenting cells (APCs) incubated with a fusion protein of rat prostatic acid phosphatase (PAP) linked to rat granulo- cyte-macrophage colony-stimulating factor (GM-CSF). This led to the first human studies of sipuleucel-T, in which prostate cancer patients treated with autologous peripheral blood mononuclear cells (PBMCs) containing APCs incubated with PA2024, the recombinant fusion protein of human PAP-GM-CSF, demonstrated immune responses to the immunizing antigen. There were no dose limiting toxicities, and PSA responses were observed in $10-15 \%$ of patients. ${ }^{1,2}$

Sipuleucel-T is manufactured from PBMCs isolated during leukapheresis procedures at weeks 0,2 , and 4. PBMCs are cultured ex vivo with PA2024. In three randomized Phase III trials of sipuleucel-T (IMPACT, D9901, and D9902A), control patients received a product made without PA2024 culture. Clinical trials were conducted in accordance with the principles of the Declaration of Helsinki. Institutional review boards approved the protocol at each study center, and all patients signed institutional review board-approved informed consent forms.

In the Phase III IMPACT trial $(\mathrm{N}=512)$, eligibility criteria included asymptomatic or minimally 
symptomatic mCRPC, castrate level of testosterone (< $50 \mathrm{ng} / \mathrm{dL}$ ) achieved via medical or surgical castration, and adequate hematologic, renal, and liver function. Baseline demographic characteristics demonstrated similar prognostic features in both arms. ${ }^{3}$

In the IMPACT study, patients randomized to sipuleucel-T demonstrated a 4.1-month improvement in median survival (hazard ratio [HR] $=0.78$ [95\% confidence interval $\{\mathrm{CI}\}$ : 0.61, 0.98]; $\mathrm{P}=0.03$ ) compared with patients randomized to control (25.8 months in the sipuleucel-T arm vs 21.7 months in the control arm). ${ }^{3} \quad$ A previous, smaller randomized Phase III study (D9901) demonstrated similar results, with a median 4.5-month improvement in overall survival $(\mathrm{HR}=0.59$ [95\% CI: 0.39, 0.88]; $\mathrm{P}=0.01)$ for patients randomized to sipuleucel-T. ${ }^{4}$ A third randomized Phase III study (D9902A), in which enrollment was prematurely discontinued, demonstrated a trend towards improved survival in sipuleucel-T patients that was not statistically significant. ${ }^{5}$ The time to objective disease progression did not differ significantly between the 2 groups in these Phase III studies.

In the IMPACT study, adverse events more commonly reported in the sipuleucel-T group (by $\geq$ $5 \%$ of sipuleucel-T patients and at least twice as frequently as control) were chills, pyrexia, headache, influenza-like illness, myalgia, hypertension, hyperhidrosis, and groin pain. ${ }^{3}$ These events were generally mild or moderate in severity and generally resolved within 1 to 2 days.

Prior to infusion, all lots of sipuleucel-T and control were evaluated for APC activation by assessing CD54 upregulation, CD54 ${ }^{+}$cell count, and total nucleated cell (TNC) count. Previous data have shown that CD54 upregulation correlates with APC activation. ${ }^{6}$ APC activation as evidenced by CD54 upregulation was evident in sipuleucel- $\mathrm{T}$, but not in control patients. In the IMPACT study, APC activation as measured by CD54 upregulation increased from week 0 to week 2 , and persisted at the third dose.

In the IMPACT study, culture supernatants from sipuleucel-T or control were evaluated for the production of APC and T cell activation-associated cytokines by multiplex luminex assay. An analysis of the culture supernatant at each week showed increases in both APC activation-associated cytokines (IL-1a, IL-1 $\beta$, IL-10, IL-12p70, IL-23, IFN $\gamma$, and TNFa) and T cell activation-associated cytokines (IL-2, IL-4, IL-5, IL-10, IL-17, IFN $\gamma$, and TNFa). ${ }^{7}$

Serum and PBMCs were obtained at baseline and at weeks 6, 14, and 26 and cryopreserved. Cellular responses were assessed by IFN $\gamma$ ELISPOT and ${ }^{3} \mathrm{H}$-thymidine $\mathrm{T}$ cell proliferation assays against PA2024 and PAP antigens while humoral responses were assessed by ELISA. The proportion of subjects with PA2024-specific IFNY ELISPOT responses $(>10$ spots) increased after treatment with sipuleucel-T. T cell proliferative responses increased from week 0 to week 6 and remained above week 0 levels at week 26 . A positive antibody response to PA2024 (antibody titer $>400$ by ELISA) was observed in $66.2 \%$ of patients treated with sipuleucel-T and $2.9 \%$ of patients treated with control. ${ }^{3}$ Positive antibody responses to PAP were observed in $28.5 \%$ of patients treated with sipuleucel-T and $1.4 \%$ of patients treated with control. ${ }^{3}$ Anti-PA2024 and anti-PAP titers increased from baseline to week 6 for patients in the sipuleucel-T group, but not among patients in the control group.

In summary, sipuleucel-T elicits antigen-specific memory $\mathrm{T}$ cell responses and humoral responses that are long-lasting. The pattern of APC activation is consistent with priming by the first infusion, and boosting by the second and third infusions. As the first autologous cellular immunotherapy to demonstrate an improvement in overall survival in asymptomatic or minimally symptomatic mCRPC patients, sipuleucel-T represents a new treatment paradigm in oncology.

\section{Abbreviations}

APC: antigen presenting cell; CI: confidence interval; GM-CSF: granulocyte-macrophage colony-stimulating factor; HR: hazard ratio; mCRPC: metastatic castrate resistant prostate cancer; PAP: prostatic acid phosphatase; PBMC: peripheral blood mononuclear cell; PSA: prostate specific antigen; TNC: total nucleated cell.

\section{Acknowledgements}

The author wishes to thank the patients and their families, the participating study sites, the clinical investigators, and the contributions of current and former Dendreon personnel in the conduct of these clinical studies. Kim Miller provided writing assistance in the preparation of this manuscript.

\section{Conflict of Interest}

The author has declared that no conflict of interest exists.

\section{References}

1 Burch PA, Breen JK, Buckner JC, et al. Priming tissue-specific cellular immunity in a phase I trial of autologous dendritic cells for prostate cancer. Clin Cancer Res. 2000 Jun;6(6):2175-82.

2 Small EJ, Fratesi P, Reese DM, et al. Immunotherapy of hormone-refractory prostate cancer with antigen-loaded dendritic cells. J Clin Oncol. 2000 Dec 1;18(23):3894-903.

3 Kantoff PW, Higano CS, Shore ND, et al. Sipuleucel-T immunotherapy for castration-resistant prostate cancer. N Engl J Med 2010;363(5):411-22. 
4 Small EJ, Schellhammer PF, Higano CS, et al. Placebo-controlled phase III trial of immunologic therapy with sipuleucel-T (APC8015) in patients with metastatic, asymptomatic hormone refractory prostate cancer. J Clin Oncol. 2006 Jul 1;24(19):3089-94.

5 Higano CS, Schellhammer PF, Small EJ, et al. Integrated data from 2 randomized, double-blind, placebo-controlled, Phase 3 trials of active cellular immunotherapy with sipuleucel-T in advanced prostate cancer. Cancer. 2009 Aug 15;115(16):3670-9.

6 Sheikh NA, Jones LA. CD54 is a surrogate marker of antigen presenting cell activation. Cancer Immunol Immunother. 2008 Sep;57(9):1381-90.

7 Sheikh NA, dela Rosa C, Frohlich MW, et al. Sipuleucel-T treatment results in sequential ex vivo activation of APCs and T cells during the culture step - evidence for in vivo immunological priming. Washington DC: Annual Meeting of the American Association for Cancer Research; 2010. 